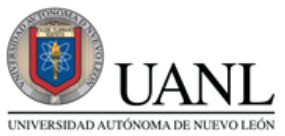

FACPYA
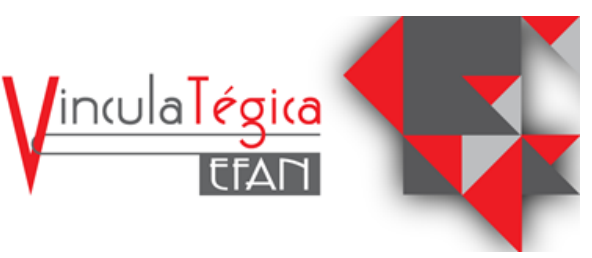

\title{
Del dinero fiat a las stablecoins. Una reflexión financiera
}

\author{
Miguel Ángel González Romero ${ }^{1}$, Sergio Gabriel Ordoñez Sánchez ${ }^{2}$ y Gerardo Ablanedo Rosas ${ }^{3}$ \\ ${ }^{1}$ Benemérita Universidad Autónoma de Puebla, Facultad de Contaduría Pública, Puebla, México, correo: \\ miguel.gonzalez@correo.buap.mx, Av. Universidad No.77 Col. Ciudad Universitaria, Teléfono 22222955 \\ 00 ext. 7670 \\ ${ }^{2}$ Benemérita Universidad Autónoma de Puebla, Facultad de Contaduría Pública, Puebla, México, correo: \\ sergio.ordonez@correo.buap.mx, Av.Universidad No.77 Col.Ciudad Universitaria, Teléfono 22222955 \\ 00 ext. 7670 \\ ${ }^{3}$ Benemérita Universidad Autónoma de Puebla, Facultad de Contaduría Pública, Puebla, México, correo: \\ Gerardo.ablanedo@correo.buap.mx, Av.Universidad No. 77 Col. Ciudad Universitaria,Teléfono 222229
} 5500 ext. 7670

Información del artículo revisado por pares

Fecha de aceptación: junio-2021

Fecha de publicación en línea: diciembre-2021

DOI: https://doi.org/10.29105/vtga7.1-108

\section{Resumen:}

La tecnología asociada a las finanzas ha penetrado fuertemente las actividades $\mathrm{y}$ transacciones económicas del mundo, esto aunado a la denominada globalización financiera que se diera en la década de los 70's ha permitido repensar el papel que juega el Estado en cuanto a la creación circulación y regulación centralizada del dinero.

De este modo, a partir de una revisión de las teorías representativas sobre la discusión del dinero fiat, este trabajo ha permitido generar una contextualización sobre la transformación del dinero en su forma tradicional de circulación a la formación de las denominadas stablecoins. De tal forma que el análisis cualitativo para abordar la realidad como hechos históricos que transforman la conducta social, es que se vislumbra el reto que tiene el Estado frente a la disyuntiva de aceptar que las finanzas descentralizadas es hoy una opción más que viable y con ello poner en jaque el ideal de la política monetaria o bien, no asumir que el rol que hoy juegan las finanzas y la tecnología unidas puede ser un vehículo que avance cada vez más rápido siendo la opción más viable del mundo moderno.

Palabras clave: Fintech, cripto activos, cadena de bloques, stablecoins.

\begin{abstract}
:
The technology associated with finance has strongly penetrated the world's economic activities and transactions, this together with the so-called financial globalization that occurred in the 70's has allowed to rethink the role played by the State in terms of the creation of circulation and centralized regulation of money. Thus, based on a review of the representative theories on the discussion of fiat money, this paper has provide a contextualization of the transformation of money from its traditional form of circulation to the formation of the so-called stablecoins. In such a way that the qualitative analysis to address the reality as historical facts that transform social behavior, is that the challenge that the State has to face the dilemma of accepting that decentralized finance is today a more than viable option and thus putting in check the ideal of monetary policy or not to assume that the role played today by finance and technology together can be a vehicle that advances faster and faster being the most viable option in the modern world.
\end{abstract}

Keywords: Fintech, cryptoassets, blockchain, stablecoins.

JEL codes: G1, G29, E4, E5 


\section{INTRODUCCIÓN}

Este trabajo forma parte de los avances de una investigación y observación más profunda que como cuerpo académico y grupo de investigación se desarrolla al interior de la Facultad de Contaduría Pública de la Benemérita Universidad Autónoma de Puebla (BUAP) y de los hallazgos en el proceso de investigación doctoral de uno de sus autores.

El Estado como ente rector de la política económica también articula la política monetaria constituyendo al Banco central como el garante de la impresión, circulación y control de la masa monetaria y el reconocimiento de los instrumentos que deben ser considerados, aceptados e intercambiados como vehículos de valor monetario, considerados dinero. En ese sentido, es el Estado quien da validez a los instrumentos que pueden ser utilizados como medios de intercambio en un mercado regulado, organizado y supervisado por el propio Estado.

Sin embargo, la creciente evolución tecnológica aunada a las finanzas ha hecho que se desarrollen herramientas financieras que permitan la realización de transacciones alternas al mercado tradicional ${ }^{1}$, lo que no sólo

${ }^{1}$ Al referirnos a las transacciones tradicionales, lo hacemos para denominar las transacciones mercantiles que permiten el intercambio de ha generado una ola de especulación sobre los activos virtuales que en estas transacciones se utilizan, sino la formación de una burbuja financiera que a partir de la desregulación se alimenta y se acrecienta. No obstante, estas consideraciones, algunas economías a nivel internacional han observado que la tecnología aplicada a las operaciones financieras podría ser reguladas y retomadas por el Estado para crear nuevos activos financieros que permitan la formación de una nueva moneda de uso corriente y reconocida como medio de intercambio en las operaciones propias de los mercados tradicionales, lo que ocasionaría una transformación histórica en el papel del Estado frente a la política monetaria.

\section{MARCO TEÓRICO}

\subsection{El dinero y su transformación histórica}

El dinero es la representación social del valor, siendo el Estado el que lo establece, pero el contexto histórico-social el que lo reconoce y da una medida de valor que es lo que le permite entonces ser concebido como una mercancía más, es decir, a partir del reconocimiento del dinero como mercancía es que el valor se objetiviza, descartando de esa manera la subjetividad y complicaciones

mercancía disponibles en el mercado a través de moneda de uso legal (monedas, billetes tanto de manera física como a través de medios digitales) 
propias de los primeros albores del intercambio a partir del trueque. Arthur (2016 p.45) afirma que el dinero es una representación de algo dado en las mercancías, sin la única manera de hacer presente al valor (...); el dinero es la actualidad del valor. ${ }^{2} \mathrm{~A}$ esto Escorcia (2016, p. 63) añade que el dinero es una creación del Estado como autoridad. Así, en tanto que este tipo de instrumentos tiene la validez estatal, pueden ser usados para la realización de transacciones con independencia de su valor intrínseco y de su valor de cambio.

Así, los mercados abiertos de capital se vuelven vehículos para la actividad especulativa, fenómenos como los ocurridos en la crisis de 2008, las burbujas en los mercados de capitales, los mecanismos de cobertura, la apuesta por el tipo de cambio o el aumento del precio de los bienes inmuebles dan testimonio de este proceso meramente basado en la esperanza de compartir los beneficios que llegue a obtener el depositario del excedente de capital puesto en estos procesos.

\subsection{Globalización financiera}

${ }^{2}$ Para Arthur, el valor se define como un "poder de intercambiabilidad"; a diferencia de Marx en quien el valor surge a partir de la extracción de la plusvalía, producto de la fuerza de trabajo impresa en las mercancías, sin embargo, si bien Marx afirma que el valor es el resultado de la extracción de la mano de obra, no es hasta que la materia prima se transforma en mercancía y está entra en circulación en el mercado cuando entonces el valor de la misma se objetiviza, esto es, a partir del
Los cambios que sufrió el sistema capitalista en la década de los 70's, comienzan por la supresión de los acuerdos de Bretton Woods (1944-1971), el auge del sistema de producción Fordista, la politización de las finanzas, la crisis petrolera de 1973, la caída del modelo de acumulación y consumo de masas permitiría la formación de un nuevo modelo $^{3}$ "que diera pasó de la manufacturación de bienes a la fabricación de ideas y al tratamiento de la información, no del personal ni de las ideas" (García, 2010 p.83).

Así, el auge de las finanzas en los procesos económicos, políticos y sociales han establecido nuevas formas de interacción en la generación de valor, ya Marx hablaba del papel de las finanzas en cuanto a la forma en la que la producción podría verse incrementada a partir de recurrir al capital que devenga interés como elemento fundamental del crédito, lo que, según el autor de El Capital, sería la razón fundamental de los bancos. Sin embargo, esta tesis ha sido causa de un sin fin de estudios que orillan a pensar que en el propio Marx existe una dialéctica en la que las finanzas no sólo están involucradas

intercambio es cuando el valor hecho mercancía se traduce en dinero incrementado, lo que conlleva a la recuperación de la inversión que hace el capitalista en los medios de producción más una plusvalía traducido en dinero incrementado ( $D^{\prime}$ ), por ello Arthur, sintetiza el proceso capitalista de producción e intercambio en la simplificación de un poder de intercambiabilidad.

${ }^{3}$ Ver Arrighi, 2000 y Stiglitz, 2003. 
en el papel del desarrollo bancario en la formación de créditos sino que además existe una perspectiva aún más compleja que tiene que ver con el hecho de poner a disposición los excedentes de capital no a merced de la producción sino que a partir de nuevos esquemas o esquemas paralelos a la generación de mercancías, el capital pueda extraer una ganancia distinta a la plusvalía, es así como a partir del concepto de capital bancario de Marx, se empieza acuñar conceptos como capital financiero, capital parasitario, capital especulativo y capital ficticio, de cuyo análisis singular y pormenorizado no habrá de dedicarse este trabajo.

\section{MÉTODO}

En una investigación cualitativa, el investigador se interesa por las personas, por su estilo y reproducción vital, sus perspectivas, historias, comportamientos, experiencias interacciones, muchos de estos intereses de corte subjetivo, pero es que la metodología cualitativa se abordan y explican bajo una lógica causal; "sin embargo, el análisis de cómo las personas ven las cosas no puede ignorar la importancia de cómo hacen las cosas" (Vasilachis, 2016 p. 26), con ello, se validan cuatro de las cinco finalidades planteadas para la investigación cualitativa a partir de Maxwell (1996: p.17-20 en Vasilachis, 2016) pues el trabajo que se desarrolla en este documento pretende comprender el contexto particular en el que los participantes del proceso de transición del dinero fiat al dinero como valor representado por las stablecoins participan, a fin de identificar los fenómenos e influencias que existen en torno a la construcción de una teoría propia, comprender los procesos que se van generando a fin de desarrollar explicaciones causales válidas. (p.31).

En esta investigación que se presenta, se plantea un énfasis personal por observar más que experimentar, encontrar significado a los comportamientos sociales, analizar la construcción del discurso que envuelve a las plataformas en las que circulan estos activos financieros.

La omisión consiente de datos duros en este trabajo responde a verificar el comportamiento no estadístico sino en la evidencia de la construcción del discurso financiero, su imposición y transmisión a partir de los actos sociales y personales de los individuos, cuyas repercusiones seguramente serán objeto de estudio, medición e interpretación cuantitativa de otro momento de quienes hoy escribimos este texto o bien de alguien cuyo interés este enfocado en la construcción de un modelo matemático y no en la construcción del escenario social actual. 


\section{RESULTADOS}

Independientemente de la teoría sobre la creación del dinero (endógena o exógena), sobre la cual se puede basar el análisis de la generación de la representación social del valor conocida como dinero emitido por el Estado a partir de su función de regulador (teoría exógena) o del reconocimiento de la creación de valor a partir de las funciones propias de la producción y del mercado (teoría exógena), se reconoce que el valor esta contenido de manera intrínseca en la representación impresa (billetes) o acuñada (monedas) cuyo control de emisión radica en la facultad exclusiva de los bancos centrales.

Bajo esta misma lógica y de acuerdo con el Banco de México (s.f.), el dinero es un conjunto de activos de una economía que las personas regularmente están dispuestas a usar como medio de pago para comprar y vender bienes y servicios.

Esta representación social del dinero a través de la generación de billetes y monedas respaldadas por un banco central, es conocido como dinero fiat, aludiendo al vocablo latino que se traduce como "hágase". Por tanto, el dinero fiat, es producto de la decisión del Estado por emitir dinero bajo una representación social controlada.
Es precisamente el Banco de México (s.f.) quien afirma que el dinero cumple tres funciones en una economía:

1. Medio de cambio: ya que es generalmente aceptado por la sociedad para saldar la compra y venta de bienes y servicios.

2. Unidad de cuenta: porque permite fijar precios y documentar deudas.

3. Depósito de valor: debido a que posibilita transferir la capacidad para comprar bienes y servicios a lo largo del tiempo.

Sin embargo, ha sido precisamente esta función del Estado de ser el ente centralizador y regulador la que se ha puesto en tela de juicio la que ha dado la pauta para encontrar medidas alternas a este problema, mismo que a continuación se describe.

\subsection{El rol del Estado como ente regulador}

Es a partir del final de la década de los 80's, cuando se concibe un Estado reducido al mínimo, la incorporación de políticas fiscales regresivas como mecanismo de desarrollo y expansión de las economías domesticas trajeron como consecuencia una flexibilización laboral, aumento en las brechas de desigualdad acumulando mayor riqueza en la cúspide de la pirámide social y permitiendo una liberación completa del mercado lo que 
generó un caldo de cultivo para la formulación de nuevas formas de intervenir desde las finanzas en la economía, de tal forma que poco a poco el crédito como mecanismo de aumento en la producción de mercancías y con ello la posibilidad de aumentar la tasa de ganancia fueron quedando relevadas al comenzar una ola en la que el ingenio financiero fuera más importante que la optimización de la producción.

Esto ha permitido que el dinero circule fuera de la esfera productiva para pasar a un plano meramente especulativo pues "no hace falta ser más productivo para obtener beneficios, sino que es suficiente con jugar en los mercados bursátiles" (Bourdieu, 1998, 2002, en García, 2010 p.86). Este proceso en el que las finanzas se incorporan con mayor fuerza en los procesos capitalistas, se ha acrecentado a partir de que el dinero ha aprovechado la desregulación y la liberación de los flujos de capital para construir escenarios favorables con mayor éxito a partir de la las décadas de los 80 y 90's; esto ha incentivado la especulación y desdeñando todo intento de intervencionismo del Estado a partir de la política monetaria como lo plantearía la escuela de Keynes y los post keynesianos al afirmar que el dinero se genera

\footnotetext{
${ }^{4}$ Ver planteamiento de Duménil, G., y Lévy. D. (2014). La grande bifurcación. En finir avec le néolibéralisme, París, La Découverte.
}

a vía una autoridad (chartalismo) (Escorcia y Robles, 2016 p. 31).

Es entonces a partir de estos planteamientos que se comienza a formar la denominada globalización financiera que no es más que el proceso en el cual la actividad financiera sobre pasa a la actividad productiva y alienta la generación de flujos de capital que corren de manera universal a partir de la especulación, así, el auge de las estructuras financieras en el sistema capitalista ha permitido la creación de la denominada revolución gerencial o revolución de la gestión ${ }^{4}$, en el que los dueños de los capitales son desplazados por gerentes que comienzan a formar una tercera clase social que se sitúa en medio de los capitalistas y de la clase obrera. Minsky (1998 p. 5-6) que retoma Escorcia y Robles (2016 p.26) contribuye a este debate planteando que:

"los administradores del dinero están
reemplazando a los gerentes de la
industria como actores principales de la
economía [...]. El capitalismo moderno
se caracteriza por la forma corporativa
de la organización de los negocios y de
las complejas estructuras financieras
que incluyen instrumentos negociables.
Por medio de la deuda y de las
obligaciones financieras, los gerentes
corporativos controlan los activos de
capital reales de la economía: los
instrumentos financieros distribuyen las


ganancias brutas presentes y futuras de las empresas presentes y futuras".

En resumen, a decir de García (2016 p.89) y a manera de explicación de aquello que ha permitido la expansión de la Globalización financiera son "problemas de información asimétrica (Stiglitz, 2003); ciclos sistémicos de acumulación (Arrighi, 1998); expectativas irracionales de los inversores (Kindleberg, 1991); orgías especulativas (Galbraith, 1991)”.

Esto ha generado incluso una nueva forma de nombrar a estos procesos de generación de riqueza financiera frente a la generación de riqueza productiva, llamándolos financiarización, término que será utilizado para referir a los procesos de generación de riqueza mediante el uso de instituciones o estructuras financieras dentro de los mercados, pues "los capitalistas, desde la década de 1970, han reorientado su actividad central desde el área productiva a la financiera" (Wallerstein, 2010:137).

En ese sentido, para Claudio Katz (2002: 191) "algunos denominan «financiación»al nuevo marco creado por el predominio de operaciones especulativas y afirman que estas transacciones se caracterizan por vertiginosos cambios de precios divorciados del empleo de los recursos".
En este mismo orden de ideas, el Estado mexicano ha sido punta de lanza en el intento de regular la creación y circulación del dinero a través de las plataformas digitales, por ello que el 8 de marzo del 2018, se firmó la Ley Fintech en México, misma que fue publicada en el Diario Oficial de la Federación (DOF) el 9 de marzo entrando en vigor al día siguiente de su publicación.

Uno de los objetivos de dicha ley, es la del fortalecimiento de la industria Fintech e intenta regular tres aspectos importantes de la industria, las empresas de financiamiento colectivo, las instituciones de fondo de pago electrónico y las operaciones con activos virtuales. En este último caso la ley FinTech permite a las Instituciones de Tecnología Financiera ("ITFs") realizar transacciones con activos virtuales que previamente hayan sido autorizados por el Banco de México.

Se espera que, eventualmente, la regulación secundaria que será emitida por Banco de México determine el alcance de la supervisión, así como los requisitos para la aprobación de activos virtuales y/o en su caso, el período para que Banco de México emita una lista de las criptomonedas autorizadas y prohibidas en México.

Aunado a esta regulación, el Instituto Mexicano de Contadores Públicos (IMCP) publicó la Norma de Información Financiera 
(NIF) C-22 cuya entrada en vigor fue el 1 de enero de 2021, con la finalidad de valuar el resultado obtenido en la operaciones con activos virtuales, sin embargo, no es menester de este documento discutir sobre los procesos de reconocimiento y valuación con activos virtuales para mostrar su efecto contable y/o fiscal, sino el de poner énfasis en la posibilidad cada vez más concreta de que el Estado pierda su hegemonía en la creación de dinero como medio de valor para ceder el paso a la creación de dinero virtual que responde a la lógica de la tecnología de bloques y no a los lineamientos de política monetaria como lo hace el dinero fiat hoy en día.

\subsection{Capital especulativo y ficticio}

A partir de la propuesta de Marx para denominar distintas formas que adopta el capital, es que se puede encontrar que existe una forma de capital que "retoma la categoría capital ficticio para referirse a la creación de valor que ocurre fuera de la esfera productiva" (Durand, 2017 en Meireles y Villavicencio, 2019 p. 66), es así como entonces el auge de la globalización financiera permite encontrar fuera de la esfera de la producción mecanismos para extraer la ganancia, siendo así, que ahora los medios de obtención de la misma están en derivados, coberturas, paridades cambiarias e instrumentos que utilizan tecnología financiera y que circulan en los mercados principalmente bursátiles, generando un capital ficticio, que no es un capital inexistente, sino que aprovechándose de la desregulación y como lo afirman los teóricos del enfoque exógeno del dinero, es dinero que genera dinero, pero que no se materializa en mercancías ni en dinero impreso, sino que es dinero presente que genera dinero futuro con la esperanza de que en el tiempo se convierta en dinero tangible materializado y objetivizado, es decir, el capital ficticio encuentra su génesis en el capital especulativo, aquel capital que aprovechando la confianza y esperanza de los individuos pone su excedente en instrumentos de ingeniería financiera con la esperanza de generar dinero que en el futuro pueda disponer.

\subsection{Tecnología blockchain}

Así, surge entonces, una nueva oleada en el que ahora no sólo las finanzas se apoderan de los procesos económicos sino que se adiciona con inteligencia artificial que permite controlar los procesos financieros a partir de los procesos tecnológicos en la denominad cadena de bloques mejor conocida como blockchain en su denominación en idioma inglés, tecnología que cobró auge con la aparición de las monedas criptográficas cuyo máximo exponente es bitcoin, quien se ha consolidado como un vehículo de especulación y de generación de flujos de capital ficticio, de cuya discusión no es interés 
de este documento, pero que es necesaria la referencia contextual para lo que nos ocupa.

El concepto conocido como "moneda criptográfica", la cual fue descrita por primera vez en 1998 por Wei Dai en la lista de correo electrónico "cypherpunks", donde propuso la idea de un nuevo tipo de dinero que utilizara la criptografía para controlar su creación y las transacciones, en lugar de que lo hiciera una autoridad centralizada. (González, Martínez y Garrido; 2019 p. 1639)

Una criptomoneda es una moneda virtual que sirve para intercambiar bienes y servicios a través de un sistema de transacciones electrónicas sin la necesidad de un intermediario. A diferencia de otros intentos de dinero electrónico, las criptomonedas incorporan los principios de la criptografía para implementar una economía segura, anónima y descentralizada. (Daily, 2018)

Debido a que no existe una moneda o método de pago que sea $100 \%$ seguro, pues siempre hay posibilidad de robo o extravío, tanto en forma física como digital, la tecnología ha buscado apoyar los sistemas de pagos en cuanto a seguridad y rapidez, tal es el caso de los bitcoins y su sistema de blockchain, sistema con el cual los usuarios mediante su llave se reduce sistemáticamente la posibilidad de robo o extravío a diferencia de otras monedas. (González, Martínez y Garrido; 2019 p.1647)

\subsection{Stablecoins}

Como se ha presentado, las innovaciones tecnológicas aunadas a las actividades financieras han desarrollado nuevas formas de concebir al dinero y por supuesto, nuevas formas de circulación del mismo, sin embargo, no es todo lo que se ha logrado con estas innovaciones, pues incluso, uno de las grandes aportaciones es la de generar nuevos instrumentos de valor de intercambio y/o resguardo de valor especulativo que permiten a los excedentes de recursos financieros encontrar mecanismos de resguardo, de encuentro y de aseguramiento de sus tasas de ganancias en una esfera basada en la especulación y no así en la producción.

De esta manera surgen activos financieros virtuales denominadas Stablecoins, mismas que se definen como activos digitales cuyo diseño basado en la cadena de bloques como cualquier otra criptomoneda, pero con la finalidad de mantener su valor estable y sin volatilidad pues replican el valor de una moneda fiat como puede ser el dólar, el euro o incluso ya hay antecedentes de estas monedas referidas al valor del peso mexicano. 
El respaldo que tienen las stablecoins radica en tener activos financieros, dinero fiat, materias primas o incluso otros criptoactivos hasta por el monto equivalente de su valor inicial, eso implica entonces que existe un respaldo del valor inicial de la moneda digital lo que le da un piso de soporte que permite una mayor estabilidad en el precio, algo que no tienen otras criptomonedas en las que su valor radica únicamente por la especulación de oferta y demanda de la misma.

Hoy en día existe evidencia de tres stablecoins que ya circulan en el mundo:

1. BUSD, que está respaldada por el precio del dólar americano.

2. BGBP, que es el criptoactivo Inglés, cuyo precio está respaldado por la libra esterlina.

3. PXO, que ha sido la revelación del 2021 pues es el criptoactivo que respalda su valor en el peso mexicano.

Lo anterior revela el avance de esta tecnología ahora con la combinación de las monedas fiat, en ese sentido México es el país latinoamericano que más ha avanzado, no sólo en lo acelerado de entender estos procesos sino incluso en materia regulatoria.

\section{CONCLUSIONES}

La tecnología aplicada a las finanzas ha ocasionado la creciente ola de surgimiento de innovaciones denominadas Fintech, lo que ha puesto en tela de juicio el papel centralizador y regulador del Estado sobre la emisión, circulación y transacciones de monedas en un primer momento las denominadas fiat para ahora tensar la discusión sobre la creación, uso y transacciones de cripto activos que mediante el uso de la tecnología de cadena de bloques (blockchain) permitan la estabilización de las transacciones de activos financieros que no basen su valor en la especulación de la oferta y demanda sino que utilicen como base el valor que respaldan las monedas fiat que hay detrás de ellas, dando paso así a las denominadas stablecoins.

$\mathrm{Si}$ bien, blockchain se basa en garantizar el registro de transacciones, en la descentralización y colaboración que no le vendrían nada mal a las actividades financieras, gubernamentales y empresariales, y de esta forma colaborar para combatir los vicios de las instituciones tradicionales. Incluso la revolución del sector financiero e institucional podría estar basada en esta tecnología.

El reto ahora está en manos de los gobiernos del mundo entero, asumir el reto de aceptar que las finanzas descentralizadas es ahora una opción viable puede poner en jaque la estabilidad de las políticas monetarias gubernamentales, pero no aceptar esta realidad puede ser un tanto canto más peligroso pues el 
avance es cada vez mayor y más profundo que

su fracaso. Aún queda mucho por discutir, este

trabajo sólo es una muestra de lo que está

frente al mundo cuando de finanzas se habla.

\section{REFERENCIAS}

Arthur, C. (2016). Valor y dinero, en Esorcia, R y Robles M. (ed.) Dinero y Capital. UAM-ITACA. México.

Arrighi, G. (1998; 2007). La globalización, la soberanía estatal y la interminable acumulación del capital. Fernando López Castellano (comp.) Desarrollo: Crónica de un desafío permanente. Universidad de Granada.

BANXICO. (SF). Glosario de términos, definiciones, Banco de México. Recuperado el 1 de junio de 2017, de http://www.banxico.org.mx/divulgacion/glosario/glosario.html

Daily, F. (2018). ¿Que son las Criptomonedas? ¿Cómo funcionan las criptodivisas? Obtenido de https://es.dailyforex.com/forex-articles/2017/10/que-son-las-criptomonedas-10-de-octubre$2017 / 84544$

Escorcia, R. (2016). Dinero mercancía, sus representantes y los presupuestos de su relación dialéctica, en Esorcia, R y Robles M. (ed.) Dinero y Capital. UAM-ITACA. México.

Galbraith, J. K. (1991). Breve historia de la crisis financiera. Barcelona: Ariel.

García- Chico, L. (2015). Teoría del tiempo y representatividad del dinero. Del dinero real al dinero virtual. Publicado en Revista de estudios sobre justicia, Derecho y Economía (RJDE). 3 (julio-diciembre 2015), 1-108

González, M., Martínez, B. y Garrido, I. (2019) ¿Son las criptomonedas un instrumento de inversión, un medio de pago o una moneda digital? Caso Bitcoin, en Gutierrez, J. Y Mayorga, P. (ed) Los Retos de la Competitividad ante la industria 4.0. RICO, México. ISBN: 978-607-96203-0-8. Páginas 1637-1652.

Katz, C. (2012). Enigmas contemporáneos de las finanzas y la moneda. Publicado en Ciclos, 12 (23), 191-219.

Keynes, J. (1948). La teoría general del empleo

Kindleberger, Ch. P. (1991). Manías, pánicos y cracs. Historia de las crisis financieras. Barcelona: Ariel.Lenin, V. I. (2004). El imperialismo fase superior del capitalismo. México. Quinto Sol.

Mp, M. (11 de 05 de 2012). Introducción a los Instrumentos Financieros: concepto, utilidad y características. Obtenido de https://educacionbancaria.wordpress.com/2012/11/05/introduccion-a-los-instrumentosfinancieros-concepto-utilidad-y-caracteristicas/

Marx, C. (2015). El Capital. Crítica de la economía política. Libro I. México. Fondo de Cultura Económica.

Marx, C. (2015). El Capital. Crítica de la economía política. Libro II. México. Fondo de Cultura Económica.

Marx, C. (2016). El Capital. Crítica de la economía política. Libro III. México. Fondo de Cultura Económica.

Maxwell, J. (1996). Qualitative Research Design. An Interactive Approach. Thousand Oaks, California, Sage.

Meireles, M. y Villavicencio, G. (2019). Discusión teórica sobre la financiarización: marxistas, poskeynesianos y en economías subdesarrolladas. En Análisis Vol. 12 (No. 32) Enero- abril 2019, páginas 61-112. www.olafinanciera.unam.mx

Stiglitz, J. E. (2003). Los felices 90: la semilla de la destrucción. Madrid: Taurus.

— (2004). El malestar en la globalización. Madrid: Taurus.

— (2006). Cómo hacer que funcione la globalización. Madrid: Taurus.

Vasilachis de Gialdino, I. (coord.) (2006) Estrategias de investigación cualitativa. Barcelona: Gedisa. pp.23-64. 
Wallerstein, I. (2010, Marzo-abril). Structural crises. En New Left Review. 62, 133-142. 